\title{
An Efficient Big Data Analytic for Forecasts the User Behavior Bus Rapid Transit System
}

\author{
Anumolu Venkat Vardhan, Danala Venkata Revant Naidu
}

\begin{abstract}
The present paper proposes in road based mass transit system, this stage might be a solution to consider by provides quality of service. This text propose a path of predict stage for this sort of transport system. This system estimates time by acknowledging its historical behavior, diagrammatic by historical profiles, and more additionally those present conduct recorded on the overall public transport vehicle that the prediction is will be made. The model employments those k-medoids bunch algorithmic system on get historical travel chance profiles. A pertinent feature of the model may be that it doesn't necessity later period knowledge from elective vehicles. To this reason, the planned model may be also used on intercity transport contexts in which service coming up with is administrated per timetables. The fast pace of developments in computer science (AI) is providing new opportunities to boost the performance of various industries and businesses, together with the transport sector. The innovations introduced by AI embody extremely advanced procedure ways that mimic the means the human brain works.
\end{abstract}

Keywords: Period Prediction, Automatic vehicle location, Road based mass transit systems, clustering.

\section{INTRODUCTION}

Urban traffic challenge could be a highly regarded topic among planners and traffic engineers. They're trying to find solutions to guard residents from this vital drawback. Thus, they propose numerous transit systems to decrease congestion, pollution and fuel consumption. However, thanks to the shortage of enough funds in developing countries, all individuals couldn't access to the high-quality transit systems (rail and metro). Therefore, an analogous various like BRT system can be the simplest possibility for them. ITDP interprets BRT as a high quality bus-based travel framework that conveys fast snug and effective urban personal satisfaction through the supply from differentiate right-of-way infrastructure, frequent operations and excellence in promoting [1]. Currently, around twenty five million BRT users with thirty, 000 buses are existed across the world [2].

\section{LITERATURE REVIEW}

Dozens of studies are performed to research the introduction of the BRT system and its impacts. Deng et al. [9] were known that when introduction of national capital BRT Line one, 75.4 you look after bus users, $4.2 \%$ of taxi users, $1.2 \%$ of automobile commuters, $1.7 \%$ of LRT riders, $7.2 \%$ of recent commuters, 5.7 of walking users and a pair of. $9 \%$ of bicycle riders are attracted by the BRT system. Deng et al. [7] expresses that BRT idea is adopted as a key purpose for mitigation of traffic issues in national capital. What is more, Lisa et al. [10] expressed that, since the implementation of BRT in Orange Line, the traffic flow throughout the height hours in southward has been improved around seven try to traffic crowd starts eleven minutes later. When the implementation of the BRT system in geographical region, the quantity of passengers United Nations agency transported by BRT system, was comparable LRT users [19]. BRT could be a new idea in developing cities. Therefore, higher to know the individual's behaviors and their angle [11].Satiennam et al. [12] were assessed that however commuters attract to the BRT system from bike and traveler cars. Eventually, the authors by victimization modal split model and SP survey worked out that period and travel price have a big influence on selection mode. What is more, they detected that BRT will attract users from a railroad car. Whereas the quantity of motorbike users united Nations agency inquisitive about the BRT system was above the automobile users.

The Attractiveness of passengers for a replacement transit system may be the foremost vital issue for the success of a replacement transit system and wishes to be thought of fastidiously. Currie et al. [13] examined travelers attractiveness by victimization trip attributes between BRT, bus, lightweight rail and serious rail, finally the analysis specific that every one the systems ar favored relative bus system. The finding conferred that BRT is extremely effective sort of a lightweight rail and serious rail, however BRT system has additional advantage in term of price. What is more, age, gender, period, travel price, menage size and financial gain ara elementary parameters in term of mode selection.

Very little study has been handled on introducing of recent transit system or overall public transit just in case of national capital town. Jalal et al. [14] when analysis of travel demand modeling were reportable that national capital town road network has enough capability to manage this vehicles except for reduction of congestion, implementation of the high capability transport modes is needed. Similarly, Noori [15] was conducted a study relating to challenges of traffic in national capital town finally the analysis has been counseled improvement of the bus network, increasing of bus terminals and introducing of the mass mass rapid transit system. In summation, may be expressed that BRT system could be a property transport system to mitigate urban transport challenges. Additionally, the quantity of users United Nations agency is interested to shift from alternative modes to the BRT system depends on the native circumstances, travel options and level of service. 


\section{IMPLEMENTATION}

\subsection{Data and Methodology}

Data achieved from completely different sources. notably, the most data for estimation of mixture travel demand obtained from national capital programmer that the survey has been accomplished by the Japan International Cooperation Agency. Supported JICA data in 2009 the quantity of population was calculable at four.01 million and five hundred,000 households existed among town. The survey solely was lined five, 000 households that represents 1 Chronicles of the overall population. For estimation of travel demand, the data from the year (2009) is taken into account as a base then the demand forecasts for the horizon year (2018). Finally, the BRT route known supported high traveler demand. The second step for understanding of the factors that ar affected on modal shift starts when the definition of the passageway for the purposed BRT system. The SP survey has been administrated on the passageway that is specific through estimation of travel demand modeling.

\subsection{Travel Demand Modeling}

This section provides the travel demand modeling method. Choice of route for transit system includes a profound impact on current and future development of town. During this study travel demand is one in every of the many determiner for choice BRT route. Travel demand modeling consists of many theoretical steps that try to interpret passengers' behavior throughout the trip. The procedure is called four-step technique and also the method consists of steps like generation/attraction model, distribution model, mode split model and assignment.

\subsection{Trip Generation}

Trip generation is that the beginning of travel demand modeling. the target of trip generation model is to predict the overall range of journeys generated by zone $\mathrm{i}$ and drawn to zone $\mathrm{j}$. during this analysis, the multiple regression model by victimization freelance variables (population, employment, automobile possession, density) extracts trip generation and attraction in twenty two districts of national capital town. the subsequent equations are wont to approach trip generation values.

\subsection{Trip Distribution}

Trip distribution is that the second step of travel demand modeling that is employed to know what percentage journeys distribute from one explicit traffic zone to a different zone. Gravity model is employed to estimate trip distribution. The gravity model is extremely common among planners and traffic engineers since it planned in 1950 and considerably improved in 1960 .

\subsection{Mode Split Model}

The modal split may be calculable supported mixture models by victimization zonal data and may be calculable through disaggregate models by utilization of menage characteristic. Modal split model assesses the range of journeys on numerous modes between distinct traffic zones. Growth Curve perform equation is employed to specify the quantity of users in existing modes. the typical speed for existing modes obtained through conversion technique by having average period for every mode and minimum route distance.
Our planned to create the individuals to travel within the route peacefully and with none interrupt in it. Which create the trail with shorter distance and with none interrupt to individuals to succeed in the destination. This is often measured by taking a reference space on the route over a brief amount of your time. In watch, it's deliberate by the work of circle finders and it's wont to compute the time taken to the vehicles to prevail in subsequent rush hour gridlock sign and furthermore to figure the time once the traffic will be clear. The decision that chooses the time the traffic stream has been finished by Artificial Neural Network. By exploitation the Back-Propagation algorithmic program to ascertain the specific time taken for the vehicles to cross the sign. This ANN is utilized for recognizing the example for the progression of traffic to anticipate the specific outcome. This may investigate the ceaseless progression of the vehicles and also for the expectation of future control framework. Which on the off chance that anybody of the course has out of reach interfere with it totally was hinted to the client and makes them to require other courses to prevail in the goal. It helps the vehicle, genuine vehicles, and autos to have an expressway and make the overall population vehicles like transports to move inside the unequivocal. The Prediction of the control the information's gathered by cameras and sensors are hang on inside the denotive information base that is valuable in foreseeing the traffic all through the stature hours like Monday - Fri and weekday - Sunday. The constant stream inside the traffic ar observed and hangs on in 3 totally various databases. What's more, acquiring the information from the each information and examination them with the verifiable information's and anticipate the more drawn out term stream of traffic inside the client required area. The Notifications was suggested to the client with the GPS framework and furthermore through the Mobile applications. To ascertain the traffic stream to subtleties of the vehicles and furthermore the speed of the vehicles ar determined for each [*fr1] partner degree hour. To ascertain the traffic stream in anybody course the progression of all the other way ought to try and be determined. For that, all the client manners by which are observed exploitation the wellbeing cameras and furthermore the utilized for see the speed of the vehicles. The edge perform of the units was changed to be a play out that is constant result, the Sigmoid perform. The employ of the sigmoid perform will be to give acceptable the extra information essential to the organize on execute those back-propagation honing algorithmic system. Back-propagation is acted will finding the square lapse (the slip function) of the complete network, then calculative those lapse term for those each of the yield and concealed units by exploitation those yield from the past substantial cell layer. The weights of the finish organize were after that balanced for reliance on the slip haul and likewise those provided for Taking in rate. Honing proceeds inside the honing situated till the lapse performs achieves accurate least criteria. Though the base may be prepared a really secondary criteria, the organize won't be primed should legitimately arrange a example. However whether the base is prepared excessively awful low criteria then that system could bring challenges to classifying glaring designs.

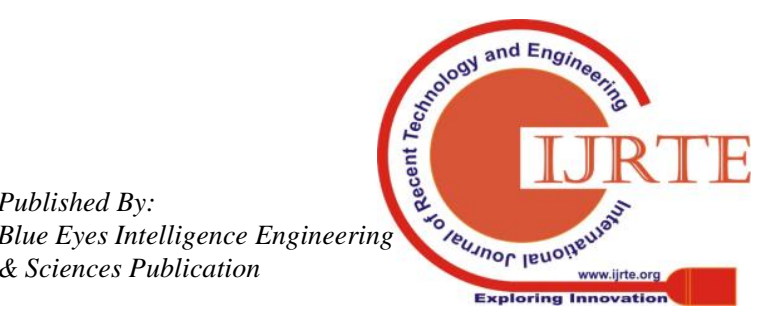




\section{RESULTS}

\section{Bus Rapid Transit}
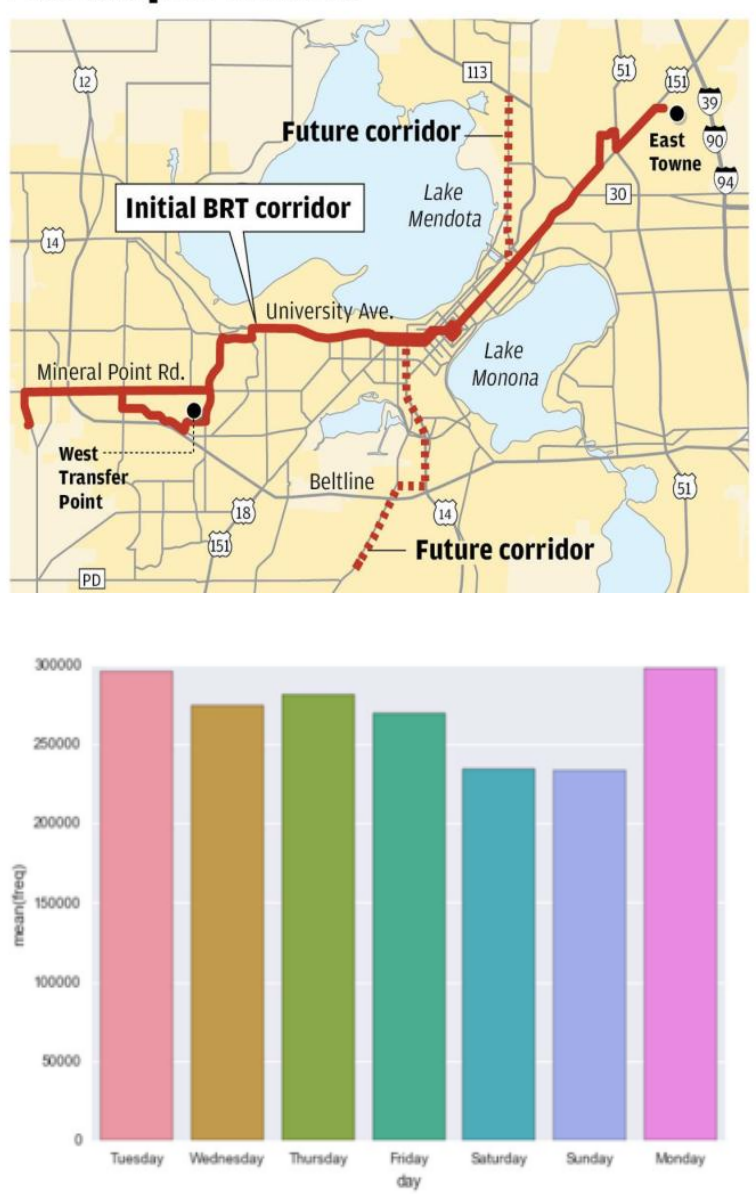

\section{CONCLUSION}

From our project the expectation of the progression of traffic has been determined before and that it will be useful for the individuals to deal with the future traffic that was made by this high populace world. We recommended that limiting the progression of vehicles will diminish the nonstop traffic that was happed in the specific region or spot which let different people groups to move rapidly from that spot. The Prediction procedure is refreshed constant will lead the adjustment in the guide consistently where the client will mindful of where they need to go. From our strict research we displayed that the usage of this working procedure was not full filled by any nations. On the off chance that it was executed in any metro urban areas it will lead a fast change in the progression of traffic in urban areas. For the future work, the traffic sign must be executed by the Intelligent Traffic System (ITS). So as to ascertain the progression of vehicles in the forecast procedure. What's more, the information base must be improved to store the huge measure of information which contains all the video, pictures and other graphical components need to store in that database.

\section{REFERENCES}

1. T.Y. Wan, "Application of conditional demand analysis in origin destination (OD) matrix estimation using traffic counts and zonalcharacteristics," Master thesis of City University of Hong Kong, 2004
2. T. V. Mathew, K. V. K. Rao, "Introduction to Transportation Engineering," National Programme on Technology Enhanced Learning, India, 2007

3. R. Cervero, "Bus Rapid Transit (BRT): An Efficient and Competitive Mode of Public Transport," Proceedings, 20th ACEA, Scientific Advisory Group Report 20, Belgium, 2013

4. Badan Pusat Statistik Provinsi DKI Jakarta, Transportation Statistics of

DKI Jakarta, Jakarta: BPS Provinsi DKI Jakarta, 2015.

5. E. Nasiboglu, U. Kuvvetli, M. Ozkilcik and U. Eliiyi, "Origin Destination Matrix Generation Using Smart Card Data: Case Study for

Izmir," 2012 IV International Conference "Problems of Cybernetics and Informatics" (PCI), Baku, 2012, pp. 1-4

6. L. X. Gao, G. y. Li, J. h. Hu and J. x. Liang, "A Calculation Method of OD Matrix in Multi-Modal Transit Network Based on Traffic Big Data," 2015 International Conference on Transportation Information and Safety (ICTIS), Wuhan, 2015, pp. 295-298.

7. W. Wang, "Bus Passenger Origin Destination Estimation and Travel Behavior Using Automated Data Collection System in London, The UK”, 2011 